\title{
Chapter 27 \\ Development of Upright CT and Its Initial Evaluation: Effect of Gravity on Human Body and Potential Clinical Application
}

\author{
Masahiro Jinzaki
}

\subsection{X-Ray Imaging of the Human Body}

The first images of the human body's interior date back to 1895, when Roentgen discovered X-rays (Röntgen 1896). An X-ray of his wife's hand provided the first image of a human internal structure. Unfortunately, the contrast produced by X-rays was not sufficient to visualize internal organs, such as the liver, spleen, kidney, or urinary bladder (Fig. 27.1).

To compensate for the weakness of low-contrast X-rays, a contrast material was developed in 1896, 1 year after the discovery of X-rays (Haschek 1896). Thereafter, iodinated contrast material (CM) was developed and has been widely used ever since (Brooks 1924; Wallingford 1953). CM enables the visualization of the vascular system, urinary tract system, biliary tract system and alimentary tract system during projection imaging using X-rays (Fig. 27.2).

\subsection{Cross-Sectional Imaging of Human Body}

In 1972, Hounsfield developed a technology called computed tomography (CT) that enabled cross-sectional imaging in humans (Hounsfield 1973). Since then, the scanning time has improved with the development of single-helical scans in 1990 (Kalender et al. 1990) and a four-row detector CT in 1999 (Hu 1999), and the scanning time was drastically improved by the development of a 64-detector CT in 2004. This development also enabled thinner slice images with a slice thickness of less

M. Jinzaki ( $\square)$

Department of Diagnostic Radiology, Keio University, School of Medicine,

Shinjuku, Tokyo, Japan

e-mail: jinzaki@rad.med.keio.ac.jp 
a)

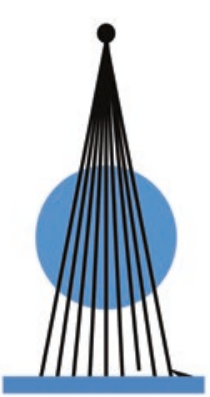

b)

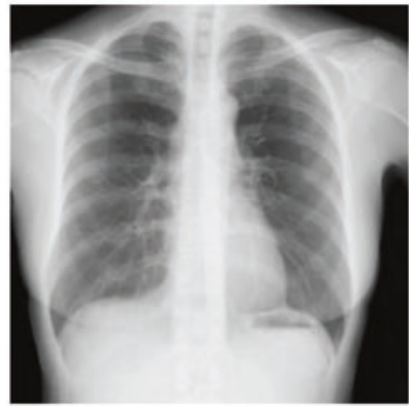

c)

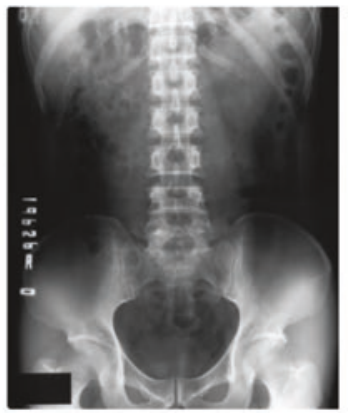

Fig. 27.1 Scheme of X-ray and X-ray images. (a) Scheme of X-ray (b) Chest X-ray images (c) Abdominal X-ray image

An X-ray image is a form of projection image. Unfortunately, the contrast of X-ray images is insufficient to visualize internal organs such as the mediastinum, liver, spleen, kidney, and bladder
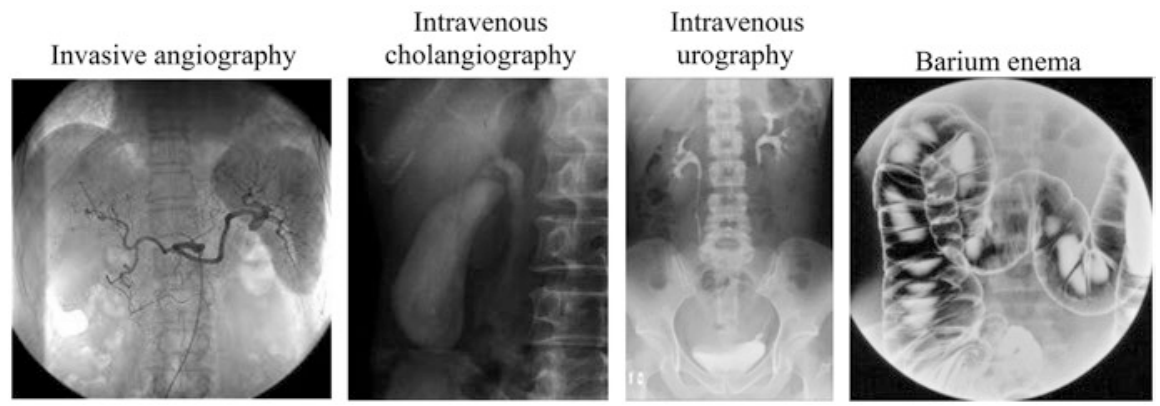

Fig. 27.2 X-ray images with contrast material

than $2 \mathrm{~mm}$ to be routinely obtained in clinical medicine. In turn, these thinner slice images enabled more detailed three-dimensional (3D) images to be created. 3D CT imaging has since replaced X-ray examinations using CM (Fig. 27.3). For example, CT angiography has replaced invasive angiography (Jinzaki et al. 2009), CT urography has replaced intravenous urography (McTavish 2002; Jinzaki et al. 2011), CT cholangiography has replaced intravenous cholangiography, and CT colonography has replaced Barium enemas (Halligan et al. 2013). These replacements have not only enabled less invasive imaging procedures, but have also improved the efficacies of diagnostic algorithms. CT examination alone, which provides both crosssectional images and 3D images, can potentially replace multiple examinations that are often required for evaluation purposes. We have devoted considerable time studying the replacement of X-ray examinations requiring $\mathrm{CM}$ with the application of 3D CT examinations (Jinzaki et al. 2009; McTavish 2002; Jinzaki et al. 2011).

Although X-ray examinations using CM have been replaced by 3D CT, simple $\mathrm{X}$-ray examinations remain widely used for two reasons. One reason is that most simple X-ray examinations are performed with the subject in a standing position. Prior to 2000, several minutes were required to obtain a whole body image using 


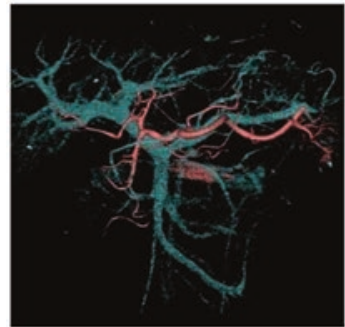

CT angiography

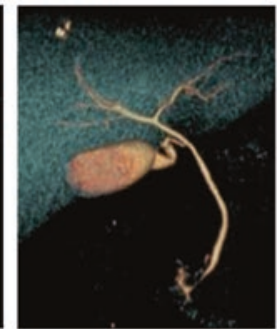

CT cholangiography

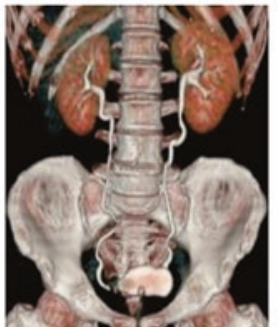

CT urography

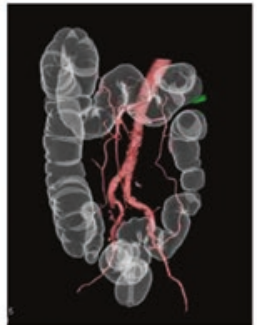

CT colonography

Fig. 27.3 Three-dimensional CT images

3D CT has replaced X-ray examinations with CM
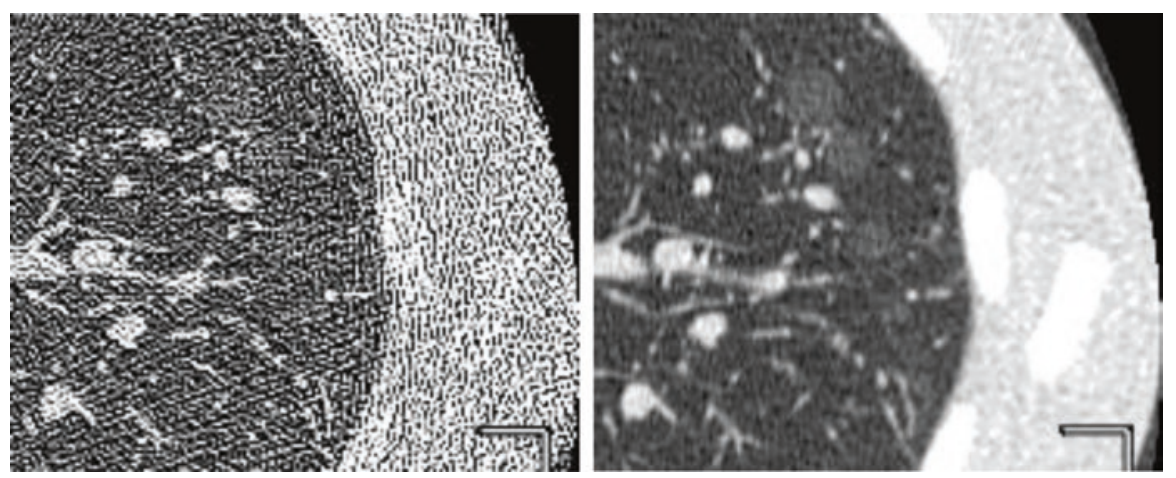

Fig. 27.4 Improvement of CT image quality using iterative reconstruction techniques

Left: An image obtained using $0.12 \mathrm{mSv}$, which is almost equal or less than the dose associated with a simple chest $\mathrm{X}$-ray examination. The image quality is very low

Right: An image reconstructed from the $0.12 \mathrm{mSv}$ image using an iterative reconstruction technique. The image quality has become acceptable

CT. Slight movements of the body are inevitable with longer CT scanning times, and these movements cause motion artifacts. The second reason is that the radiation dose required during CT examinations is significantly higher than that required during a simple X-ray examination. However, with the development of a 64-detector CT in 2004, the whole body trunk can now be scanned in less than 20 s. Also, with the re-emergence of new reconstruction techniques around 2010 (Hara et al. 2009), the radiation dose required by $\mathrm{CT}$ to maintain good image quality has gradually decreased. For example, CT images obtained at a dose of $0.12 \mathrm{mSv}$, which is almost equal to or less than the dose associated with a simple chest X-ray examination, have now become acceptable in terms of image quality thanks to the use of new reconstruction techniques (Yamada et al., 2012a, b) (Fig. 27.4).

Furthermore, a 320 detector CT device was developed in 2007, enabling a longitudinal coverage of $16 \mathrm{~cm}$ in one rotation (Rybicki et al. 2008). Repetitive acquisitions at the same position using a wider range of acquisition during one rotation has enabled four dimensional (4D) imaging (Fujiwara et al. 2013; Sakamoto et al. 2015) 

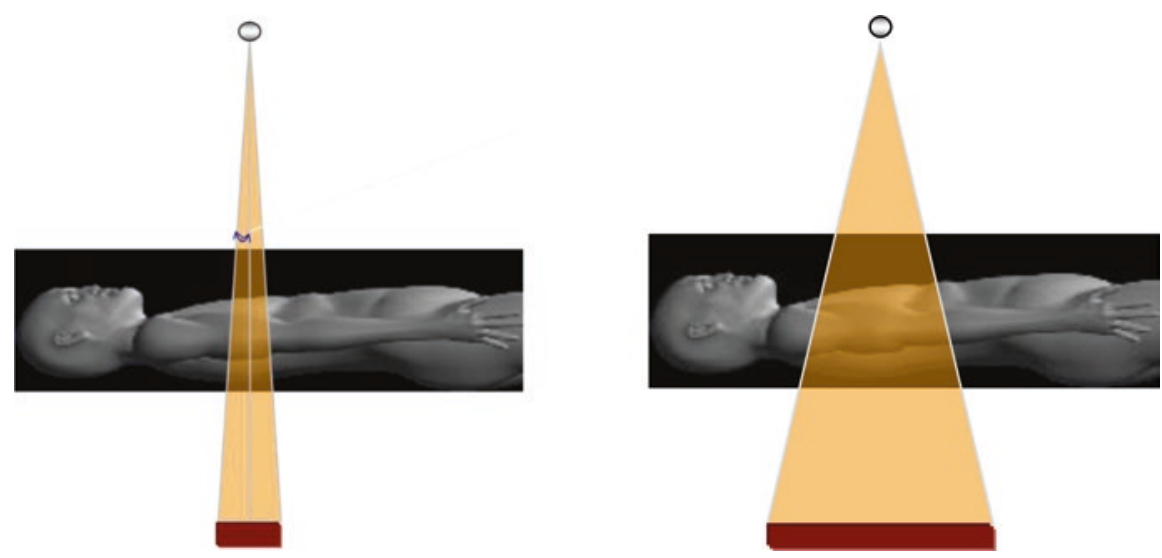

Fig. 27.5 Difference in coverage during one rotation between a 64-detector CT device and a 320-detector CT device

Left: 64-detector CT. The width of one rotation was $3.2 \mathrm{~cm}$

Right: 320-detector CT. The width of one rotation was $16 \mathrm{~cm}$

(Fig. 27.5). While 3D images, which have been used since 2004, provided mainly morphological information, the new 4D CT images can also provide functional information. However, many functions of the human body that occur with the subject in an upright position, such as swallowing, voiding, and walking, cannot be evaluated using CT machines that requiring the subject to be in a supine position.

\subsection{Development of Upright CT}

Upright CT devices offer substantial advantages: (1) they have the potential to replace poorer quality $\mathrm{X}$-ray examinations, (2) they enable the visualization of cross sections of the entire human body (including soft tissue) while the body is subjected to a load or gravity, and (3) they enable functional imaging using 4-D scanning.

We presented a proposal for an upright CT device to Toshiba Medical Systems (presently Canon Medical Systems). To assuage company concerns regarding clinical indications and profitability, we listed several conceivable clinical applications and gathered information regarding the needs and benefits of such a device from various medical fields to convince the company of the wide applications of such a device. The "Upright CT Project" was finally approved in 2014.

The most difficult task was to achieve a high-speed, high-precision vertical rotation while minimizing the vibration, since even miniscule amounts of vibration cause motion artifacts, degrading image quality. Several advanced technologies were introduced to solve this problem. The second task was to create various aids to enable the subject to maintain a stable standing position. We also created a kneehigh acrylic wall that encircles the subject's body to help prevent falls. 

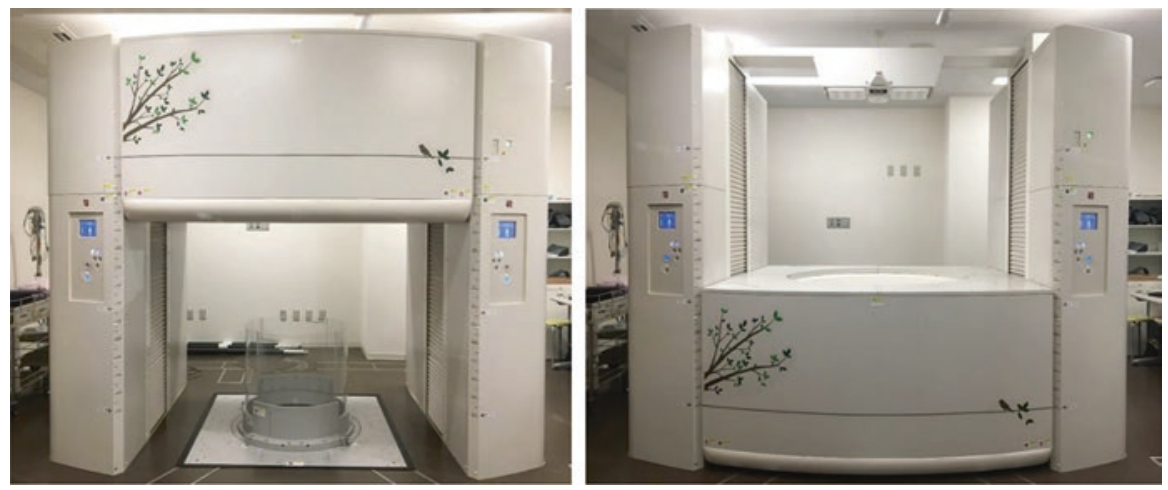

Fig. 27.6 The first upright CT machine, introduced at our institute Left: The gantry is in the up position Right: The gantry is in the down position

The resulting upright $\mathrm{CT}$ system enables vertical movements of a transverse 320 row-detector gantry (detector size, $0.5 \mathrm{~mm}$ ) with a 0.275 -s gantry rotation speed at its best performance (Fig. 27.6). This machine was approved by the Japanese Pharmaceuticals and Medical Devices Agency in March 2017.

\subsection{Physical Properties and Clinical Data Analysis}

After the introduction of an upright CT device for whole body imaging at our institute, we first evaluated several physical properties using a phantom to confirm whether the vertical movements of the gantry degraded the image quality. Spatial resolution, noise, and low contrast resolution were analyzed using a modulation transfer function, noise-power spectrum and visual inspection, respectively. As a result, each factor of the upright CT was comparable to conventional 320-detector row $\mathrm{CT}$ scanner.

During scans of volunteers, we noticed that the workflow for upright CT examinations proceeds very smoothly, compared with conventional scans. In an upright CT examination, the patient enters the CT room and proceeds directly into the gantry space; the scan begins immediately thereafter. In a conventional CT examination, however, the patient must first lie down on the scanner bed and the examiner must then raise the bed and position the bed within the gantry.

A quantitative analysis of clinical data revealed that the structure of the brain is slightly descended when the subject is in an upright position, compared with a supine position, although the brain was previously thought not to move. We also found that gravity differentially affects the volume and shape of the vena cavae depending on position, while those of the aorta remain constant regardless of the position. The lung volume was larger in an upright position than in a supine position, and the 
amount of change was larger for the lower lobe than for the upper or middle lobes. The centerline of the body was strongly correlated with the area of contact between the hip joint and the femoral head. Furthermore, we also confirmed that an upright CT examination can reveal more remarkable findings than conventional CT examinations in patients with various diseases, such as spondylolisthesis, inguinal hernia and pelvic prolapse.

We would like to quantify all human anatomical structures in three-dimensions while the subject is in an upright position and to quantify the effect of gravity on the human body by comparing CT images taken while the subject is in either a supine or upright position. Upright CT has the potential to become a powerful tool for both functional evaluations and evaluations of pathogenesis in the human body.

\section{References}

Brooks B (1924) Intra-arterial injection of sodium iodide. JAMA 82:1016

Fujiwara H, Momoshima S, Akiyama T et al (2013) Whole-brain CT digital subtraction angiography of cerebral dural arteriovenous fistula using 320-detector row CT. Neuroradiology 55:837-843

Halligan S, Wooldrage K, Dadswell E et al (2013) Computed tomographic colonography versus barium enema for diagnosis of colorectal cancer or large polyps in symptomatic patients (SIGGAR): a multicentre randomised trial. Lancet 381(9873):1185-1193

Hara AK, Paden RG, Silva AC, Kujak JL, Lawder HJ, Pavlicek W (2009) Iterative reconstruction technique for reducing body radiation dose at CT: feasibility study. AJR Am J Roentgenol 193:764-771

Haschek H, Lindenthal OT (1896) A contribution to the practical use of the photography according to Röntgen. Wien Klin Wschr 9:63

Hounsfield GN (1973) Computerized transverse axial scanning (tomography). Part 1. Description of system. Br J Radiol 46:1016-1022

Hu H (1999) Multi-slice helical CT: scan and reconstruction. Med Phys 26:5-18

Jinzaki M, Sato K, Tanami Y et al (2009) Diagnostic accuracy of angiographic view image for the detection of coronary artery stenoses by 64-detector row CT: a pilot study comparison with conventional post-processing methods and axial images alone. Circ J 73:691-698

Jinzaki M, Matsumoto K, Kikuchi E et al (2011) Comparison of CT urography and excretory urography in the detection and localization of urothelial carcinoma of the upper urinary tract. AJR Am J Roentgenol 196:1102-1109

Kalender WA, Seissler W, Klotz E, Vock P (1990) Spiral volumetric CT with single-breath-hold technique, continuous transport, and continuous scanner rotation. Radiology 176:181-183

McTavish JD, Jinzaki M, Zou KH et al (2002) Multi-detector row CT urography: comparison of strategies for depicting the normal urinary collecting system. Radiology 225:783-790

Röntgen WC (1896) On a new kind of rays. Science 3:227-231

Rybicki FJ, Otero HJ, Steigner ML et al (2008) Initial evaluation of coronary images from 320-detector row computed tomography. Int J Cardiovasc Imaging 24:535-546

Sakamoto Y, Soga S, Jinzaki M et al (2015) Evaluation of velopharyngeal closure by 4D imaging using 320-detector-row computed tomography. J Plast Reconstr Aesthet Surg 68:479-484

Wallingford VH (1953) The development of organic iodide compounds as X-ray contrast media. J Am Pharm Assoc (Scientific Edition) 42:721-728 
Yamada Y, Jinzaki M, Tanami Y et al (2012a) Model-based iterative reconstruction technique for ultralow-dose computed tomography of the lung: a pilot study. Investig Radiol 47:482-489

Yamada Y, Jinzaki M, Hosokawa T et al (2012b) Dose reduction in chest CT: comparison of the adaptive iterative dose reduction $3 \mathrm{D}$, adaptive iterative dose reduction, and filtered back projection reconstruction techniques. Eur J Radiol 81:4185-4195

Open Access This chapter is licensed under the terms of the Creative Commons Attribution 4.0 International License (http://creativecommons.org/licenses/by/4.0/), which permits use, sharing, adaptation, distribution and reproduction in any medium or format, as long as you give appropriate credit to the original author(s) and the source, provide a link to the Creative Commons licence and indicate if changes were made.

The images or other third party material in this chapter are included in the chapter's Creative Commons licence, unless indicated otherwise in a credit line to the material. If material is not included in the chapter's Creative Commons licence and your intended use is not permitted by statutory regulation or exceeds the permitted use, you will need to obtain permission directly from the copyright holder.

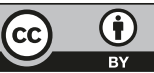

